\title{
Corrigendum: Targeting a portion of central European spider diversity for permanent preservation
}

\author{
Klemen Čandek ${ }^{\dagger}$, Matjaž Gregorič ${ }^{\dagger}$, Rok Kostanjšek ${ }^{\ddagger}$, Holger Frick§, Christian Kropfl, \\ Matjaž Kuntner ${ }^{\dagger}, \pi$ \\ † Institute of Biology, Scientific Research Centre, Slovenian Academy of Sciences and Arts, Ljubljana, Slovenia \\ ‡ Department of Biology, Biotechnical faculty, University of Ljubljana, Ljubljana, Slovenia \\ $\S$ National Collection of Natural History, Office of Environment, Vaduz, Liechtenstein \\ | Department of Invertebrates, Natural History Museum, Bern, Switzerland \\ If National Museum of Natural History, Smithsonian Institution, Washington, DC, United States of America
}

Corresponding author: Klemen Čandek (klemen.candek@gmail.com)

Academic editor: Jeremy Miller

Received: 08 Dec 2014 | Accepted: 16 Jan 2015 | Published: 21 Jan 2015

Citation: Čandek K, Gregorič M, Kostanjšek R, Frick H, Kropf C, Kuntner M (2015) Corrigendum: Targeting a portion of central European spider diversity for permanent preservation. Biodiversity Data Journal 3: e4301. doi: 10.3897/BDJ.3.e4301

After publication (Čandek et al. 2013), we discovered identification and count errors. We reexamined material and identified five species (Araniella opisthographa, Microlinyphia impigra, Oryphantes cf. angulatus, Alopecosa taeniata and Pardosa cf. saltans) that are new to the checklist and omitted eight species (Lathys nielseni, Dysdera erythrina, Megalepthyphantes nebulosus, Meioneta similis, Pelecopsis mengei, Alopecosa aculeata, Pardosa cf. hyperborea and Pellenes tripunctatus) from it. We also added cf. to sixteen less reliably identified species. All errors are corrected below in bold letters:

Abstract: The sentence with corrected counts should now read: "We here report on the faunistic part of this project, which resulted in 320 species (224 in Slovenia, 142 in Switzerland) for which identification was reasonably established."

Analysis: The paragraph with corrected counts should now read: "Altogether, we identified 1595 adult individuals, belonging to $\mathbf{3 2 0}$ species, 184 genera and 33 spider families. From the 76 localities in Slovenia, we recorded 224 species belonging to 144 genera and 31 families. From the 33 localities in Switzerland, we recorded 142 species belonging to 89 
genera and 18 families. The number of unique species was 178 and $\mathbf{9 6}$ for Slovenia and Switzerland, respectively. As an indication of rare species in our survey, singletons (those species represented on the list with a single individual (Coddington et al. 1996, Coddington et al. 2009) represented $\mathbf{3 6 . 6 \%}$ (117 species)."

\section{Checklist:}

Page 10: The entries for Araniella cucurbitina (Clerck, 1757) represent that species and Araniella opisthographa (Kulczyn'ski, 1905) (new to the checklist) and the corrected text should read:

Araniella cucurbitina (Clerck, 1757)

Materials

a. IocationID: SI41; country: Slovenia; locality: Socerb, Osp; minimumElevationInMeters: 116; maximumElevationInMeters: 116; decimalLatitude: 45.5819; decimalLongitude: 13.8558; eventDate: 2012-06-07; habitat: trail from Socerb to Osp; sex: 1 female; recordedBy: Kuntner, Gregorič, Čandek, Kralj-Fišer, Cheng

b. IocationID: SI50; country: Slovenia; locality: Sp. Prapreče; minimumElevationInMeters: 351; maximumElevationInMeters: 351; decimalLatitude: 46.1620; decimalLongitude: 14.6933; eventDate: 2010-08-03/2012-05-28; habitat: house and surroundings; sex: 1 male; recordedBy: Kuntner, Čandek

Araniella opisthographa (Kulczyn'ski, 1905)

\section{Materials}

a. IocationID: SI50; country: Slovenia; locality: Sp. Prapreče; minimumElevationInMeters: 351; maximumElevationInMeters: 351; decimalLatitude: 46.1620; decimalLongitude: 14.6933; eventDate: 2010-08-03/2012-05-28; habitat: house and surroundings; sex: 1 male; recordedBy: Kuntner, Čandek

b. IocationID: SI58; country: Slovenia; locality: Budanje; minimumElevationInMeters: 243; maximumElevationInMeters: 243; decimalLatitude: 45.8743; decimalLongitude: 13.9497; eventDate: 2011-05-07; habitat: school and surroundings; sex: 1 male; recordedBy: Čandek

Page 19-20: Due to misidentification, Lathys nielseni (Schenkel, 1932) is omitted, and the only female reported is added to the material listed for Lathys humilis (Blackwall, 1855) and the corrected text should read: 
Lathys humilis (Blackwall, 1855)

Materials

a. IocationID: SI56; country: Slovenia; locality: Dinaric Karst, Novelo; minimumElevationInMeters: 358; maximumElevationInMeters: 359; decimalLatitude: 45.8533; decimalLongitude: 13.6552; eventDate: 2011-04-04/05-10; habitat: overgrowth; sex: 1 female; recordedBy: Gregorič, Čandek, Kralj-Fišer

b. IocationID: SI41; country: Slovenia; locality: Socerb, Osp; minimumElevationInMeters: 116; maximumElevationInMeters: 116; decimalLatitude: 45.5819; decimalLongitude: 13.8558; eventDate: 2012-06-07; habitat: trail from Socerb to Osp; sex: 1 female; recordedBy: Kuntner, Gregorič, Čandek, Kralj-Fišer, Cheng

Page 20: Due to misidentification, Dysdera erythrina (Walckenaer, 1802) is omitted, and the only female reported is added to the material listed for Dysdera adriatica Kulczyn'ski, 1897 , and the corrected text should read:

Dysdera adriatica Kulczyn'ski, 1897

Materials

a. IocationID: SI12; country: Slovenia; locality: Divača; minimumElevationInMeters: 660; maximumElevationInMeters: 660; decimalLatitude: 45.6788; decimalLongitude: 14.0437; eventDate: 2012-07-22; habitat: forest; sex: 1 female, 1 male; recordedBy: Kostanjšek, RTŠB 2012

b. IocationID: SI08; country: Slovenia; locality: Ribnica, Pivka; minimumElevationInMeters: 400; maximumElevationInMeters: 400; decimalLatitude: 45.6333; decimalLongitude: 14.1392; eventDate: 2012-07-21; habitat: forest; sex: 1 female; recordedBy: Kostanjšek, RTŠB 2012

Page 23: Misspelling: Nomisia extornata should read Nomisia exornata.

Page 35, 41: Due to misidentification, Megalepthyphantes nebulosus (Sundevall, 1830) is omitted, and all representatives are added to the material listed for Mughiphantes mughi (Fickert, 1875) and the corrected text should read:

Mughiphantes mughi (Fickert, 1875)

Materials

a. locationID: $\mathrm{CH} 21$; country: Switzerland; locality: Grison Alps, Alp Flix, Salategnas; minimumElevationInMeters: 1970; maximumElevationInMeters: 1970; decimalLatitude: 46.5194; decimalLongitude: 9.6490; eventDate: 2011-07-12; habitat: swamp grazed vegetation; sex: 1 male; recordedBy: Kuntner, Gregorič, Čandek 
b. IocationID: $\mathrm{CH} 23$; country: Switzerland; locality: Grison Alps, Alp Flix, Salategnas; minimumElevationInMeters: 1900; maximumElevationInMeters: 1900; decimalLatitude: 46.5141; decimalLongitude: 9.6448; eventDate: 2011-07-12; habitat: forest opening, grass and shrubs; sex: 6 females; recordedBy: Kuntner, Gregorič, Čandek

c. locationID: CH24; country: Switzerland; locality: Grison Alps, Alp Flix, Salategnas; minimumElevationInMeters: 1830; maximumElevationInMeters: 1830; decimalLatitude: 46.5131; decimalLongitude: 9.6430; eventDate: 2011-07-12; habitat: meadow and forest; sex: 1 male; recordedBy: Kuntner, Gregorič, Čandek

d. IocationID: CH31; country: Switzerland; locality: Grison Alps, Alp Flix - Lai Neir; minimumElevationInMeters: 1910; maximumElevationInMeters: 1910; decimalLatitude: 46.5343; decimalLongitude: 9.6375; eventDate: 2011-07-16; habitat: lake and swamp around forest; sex: 5 females, 2 males; recordedBy: Kuntner, Gregorič, Čandek

e. locationID: CH32; country: Switzerland; locality: Grison Alps, Alp Flix, Salategnas; minimumElevationInMeters: 1955; maximumElevationInMeters: 1955; decimalLatitude: 46.5203; decimalLongitude: 9.6458; eventDate: 2011-07-16; habitat: timberline forest, moss; sex: 3 females, 1 male; recordedBy: Kuntner, Gregorič, Čandek

f. IocationID: CH17; country: Switzerland; locality: Engadin, Bivio; minimumElevationInMeters: 1780; maximumElevationInMeters: 1780; decimalLatitude: 46.4753; decimalLongitude: 9.6469; eventDate: 2011-07-11; habitat: forest and river edge; sex: 1 female; recordedBy: Kuntner, Gregorič, Čandek

Page 37: Meioneta similis (Kulczyn'ski, 1926) data should be deleted.

Page 38: The entries for Micrargus alpinus (O. P.-Cambridge, 1871) represent that species and Micrargus herbigradus (Blackwall, 1854) and the corrected text should read:

Micrargus alpinus (O. P.-Cambridge, 1871)

Materials

a. locationID: $\mathrm{CH} 28$; country: Switzerland; locality: Grison Alps, Alp Flix, Salategnas; minimumElevationInMeters: 1713; maximumElevationInMeters: 1713; decimalLatitude: 46.5165; decimalLongitude: 9.6387; eventDate: 2011-07-15; habitat: forest edge; sex: 1 female; recordedBy: Kuntner, Gregorič, Čandek

b. IocationID: CH30; country: Switzerland; locality: Grison Alps, Alp Flix - Lai Flix; minimumElevationInMeters: 1967; maximumElevationInMeters: 1967; decimalLatitude: 46.5358; decimalLongitude: 9.6409; eventDate: 2011-07-16; habitat: next to alpine lake; sex: 1 female; recordedBy: Kuntner, Gregorič, Čandek

c. locationID: CH31; country: Switzerland; locality: Grison Alps, Alp Flix - Lai Neir; minimumElevationInMeters: 1910; maximumElevationInMeters: 1910; decimalLatitude: 46.5343; decimalLongitude: 9.6375; eventDate: 2011-07-16; 
habitat: lake and swamp around forest; sex: 1 female; recordedBy: Kuntner, Gregorič, Čandek

Micrargus herbigradus (Blackwall, 1854)

Materials

a. IocationID: SI15; country: Slovenia; locality: Apače; minimumElevationInMeters: 220; maximumElevationInMeters: 220; decimalLatitude: 46.6804; decimalLongitude: 15.8988; eventDate: 2011-07-26; habitat: forest; sex: 1 female; recordedBy: Kostanjšek, RTŠB 2011

b. IocationID: CH03; country: Switzerland; locality: Bernese Alps, Gasteretal; minimumElevationInMeters: 1520; maximumElevationInMeters: 1520; decimalLatitude: 46.4498; decimalLongitude: 7.7135; eventDate: 2011-07-07; habitat: spruce forest; sex: 1 female; recordedBy: Kuntner, Gregorič, Čandek

Page 40, 50-51: The entries for Mughiphantes cornutus (Schenkel, 1927) represent that species and Tenuiphantes mengei (Kulczyn'ski, 1887) and the corrected text should read:

Mughiphantes cornutus (Schenkel, 1927)

Materials

a. IocationID: $\mathrm{CH} 16$; country: Switzerland; locality: Engadin, Silvaplana; minimumElevationInMeters: 1930; maximumElevationInMeters: 1930; decimalLatitude: 46.4667; decimalLongitude: 9.7946; eventDate: 2011-07-11; habitat: Larix and Pinus forest; sex: 1 female; recordedBy: Kuntner, Gregorič, Čandek

b. IocationID: $\mathrm{CH} 22$; country: Switzerland; locality: Grison Alps, Alp Flix, Salategnas; minimumElevationInMeters: 1900; maximumElevationInMeters: 1900; decimalLatitude: 46.5152; decimalLongitude: 9.6466; eventDate: 2011-07-12; habitat: forest ground; sex: 1 female, 1 male; recordedBy: Kuntner, Gregorič, Čandek

Tenuiphantes mengei (Kulczyn'ski, 1887)

Materials

a. IocationID: CH01; country: Switzerland; locality: Bernese Alps, Gasteretal; minimumElevationInMeters: 1662; maximumElevationInMeters: 1662; decimalLatitude: 46.4457; decimalLongitude: 7.7413; eventDate: 2011-07-07; habitat: alpine meadow; sex: 4 females; recordedBy: Kuntner, Gregorič, Čandek

b. IocationID: CH02; country: Switzerland; locality: Bernese Alps, Gasteretal; minimumElevationInMeters: 1698; maximumElevationInMeters: 1698; decimalLatitude: 46.4486; decimalLongitude: 7.7438; eventDate: 2011-07-07; habitat: spruce thicket and grass; sex: 14 females, 7 males; recordedBy: Kuntner, Gregorič, Čandek 
c. locationID: CH05; country: Switzerland; locality: Bernese Alps, Gasteretal; minimumElevationInMeters: 1380; maximumElevationInMeters: 1380; decimalLatitude: 46.4674; decimalLongitude: 7.6640; eventDate: 2011-07-07; habitat: river vegetation; sex: 1 female; recordedBy: Kuntner, Gregorič, Čandek

d. locationID: CH06; country: Switzerland; locality: Bernese Alps, Kandersteg; minimumElevationInMeters: 1677; maximumElevationInMeters: 1677; decimalLatitude: 46.5020; decimalLongitude: 7.6992; eventDate: 2011-07-07; habitat: alpine meadow; sex: 3 females, 2 males; recordedBy: Kuntner, Gregorič, Čandek

e. locationID: CH09; country: Switzerland; locality: Pennine Alps, Mattertal; minimumElevationInMeters: 1447; maximumElevationInMeters: 1447; decimalLatitude: 46.0976; decimalLongitude: 7.7789; eventDate: 2011-07-08; habitat: forest and meadow near river; sex: 5 females, 1 male; recordedBy: Kuntner, Gregorič, Čandek

f. locationID: CH12; country: Switzerland; locality: Bernese Alps, Nessental; minimumElevationInMeters: 930; maximumElevationInMeters: 930; decimalLatitude: 46.7213; decimalLongitude: 8.3039; eventDate: 2011-07-10; habitat: grassland and lone trees; sex: 1 female, 1 male; recordedBy: Kuntner, Gregorič, Čandek

g. locationID: $\mathrm{CH} 13$; country: Switzerland; locality: Bernese Alps, Sustenpass; minimumElevationInMeters: 2040; maximumElevationInMeters: 2040; decimalLatitude: 46.7330; decimalLongitude: 8.4324; eventDate: 2011-07-10; habitat: alpine grassland and shrubs; sex: 4 females; recordedBy: Kuntner, Gregorič, Čandek

h. locationID: $\mathrm{CH} 16$; country: Switzerland; locality: Engadin, Silvaplana; minimumElevationInMeters: 1930; maximumElevationInMeters: 1930; decimalLatitude: 46.4667; decimalLongitude: 9.7946; eventDate: 2011-07-11; habitat: Larix and Pinus forest; sex: 1 female; recordedBy: Kuntner, Gregorič, Čandek

i. locationID: $\mathrm{CH} 17$; country: Switzerland; locality: Engadin, Bivio; minimumElevationInMeters: 1780; maximumElevationInMeters: 1780; decimalLatitude: 46.4753; decimalLongitude: 9.6469; eventDate: 2011-07-11; habitat: forest and river edge; sex: 1 male; recordedBy: Kuntner, Gregorič, Čandek

j. IocationID: $\mathrm{CH} 23$; country: Switzerland; locality: Grison Alps, Alp Flix, Salategnas; minimumElevationInMeters: 1900; maximumElevationInMeters: 1900; decimalLatitude: 46.5141; decimalLongitude: 9.6448; eventDate: 2011-07-12; habitat: forest opening, grass and shrubs; sex: 2 females; recordedBy: Kuntner, Gregorič, Čandek

k. IocationID: $\mathrm{CH} 24$; country: Switzerland; locality: Grison Alps, Alp Flix, Salategnas; minimumElevationInMeters: 1830; maximumElevationInMeters: 1830; decimalLatitude: 46.5131; decimalLongitude: 9.6430; eventDate: 2011-07-12; habitat: meadow and forest; sex: 1 female, 3 males; recordedBy: Kuntner, Gregorič, Čandek

I. locationID: $\mathrm{CH} 25$; country: Switzerland; locality: Grison Alps, Alp Flix, Salategnas; minimumElevationInMeters: 1950; maximumElevationInMeters: 1950; 
decimalLatitude: 46.5159; decimalLongitude: 9.6496; eventDate: 2011-07-12/16; habitat: meadow and shrubs at stream; sex: 1 female, 1 male; recordedBy: Kuntner, Gregorič, Čandek

m. locationID: CH28; country: Switzerland; locality: Grison Alps, Alp Flix, Salategnas; minimumElevationInMeters: 1713; maximumElevationInMeters: 1713; decimalLatitude: 46.5165; decimalLongitude: 9.6387; eventDate: 2011-07-15; habitat: forest edge; sex: 3 females, 3 males; recordedBy: Kuntner, Gregorič, Čandek

n. locationID: CH30; country: Switzerland; locality: Grison Alps, Alp Flix - Lai Flix; minimumElevationInMeters: 1967; maximumElevationInMeters: 1967; decimalLatitude: 46.5358; decimalLongitude: 9.6409; eventDate: 2011-07-16; habitat: next to alpine lake; sex: 4 females, 2 males; recordedBy: Kuntner, Gregorič, Čandek

o. IocationID: CH31; country: Switzerland; locality: Grison Alps, Alp Flix - Lai Neir; minimumElevationInMeters: 1910; maximumElevationInMeters: 1910; decimalLatitude: 46.5343; decimalLongitude: 9.6375; eventDate: 2011-07-16; habitat: lake and swamp around forest; sex: 2 females, 1 male; recordedBy: Kuntner, Gregorič, Čandek

p. locationID: CH32; country: Switzerland; locality: Grison Alps, Alp Flix, Salategnas; minimumElevationInMeters: 1955; maximumElevationInMeters: 1955; decimalLatitude: 46.5203; decimalLongitude: 9.6458; eventDate: 2011-07-16; habitat: timberline forest, moss; sex: 1 male; recordedBy: Kuntner, Gregorič, Čandek

Page 42, 43: The entries for Neriene clathrata (Sundevall, 1830) and Neriene peltata (Wider, 1834) represent these species and Microlinyphia impigra (O. P.-Cambridge, 1871) (new to the checklist) and the corrected text should read:

Neriene clathrata (Sundevall, 1830)

Materials

a. IocationID: CH09; country: Switzerland; locality: Pennine Alps, Mattertal; minimumElevationInMeters: 1447; maximumElevationInMeters: 1447; decimalLatitude: 46.0976; decimalLongitude: 7.7789; eventDate: 2011-07-08; habitat: forest and meadow near river; sex: 1 female; recordedBy: Kuntner, Gregorič, Čandek

b. locationID: $\mathrm{CH} 15$; country: Switzerland; locality: Glarus Alps, near Affeier; minimumElevationInMeters: 817; maximumElevationInMeters: 817; decimalLatitude: 46.7606; decimalLongitude: 9.0933; eventDate: 2011-07-10; habitat: meadow and forest; sex: 1 female; recordedBy: Kuntner, Gregorič, Čandek

c. locationID: SI14; country: Slovenia; locality: Spodnji Velovlek; minimumElevationInMeters: 225; maximumElevationInMeters: 225; decimalLatitude: 46.4768; decimalLongitude: 15.9316; eventDate: 2011-07-25; habitat: forest; sex: 1 female; recordedBy: Kostanjšek, RTŠB 2011 
d. IocationID: SI56; country: Slovenia; locality: Dinaric Karst, Novelo; minimumElevationInMeters: 358; maximumElevationInMeters: 359; decimalLatitude: 45.8533; decimalLongitude: 13.6552; eventDate: 2011-04-04/05-10; habitat: overgrowth; sex: 1 female, 1 male; recordedBy: Gregorič, Čandek, Kralj-Fišer

Neriene peltata (Wider, 1834)

Material

a. locationID: CH02; country: Switzerland; locality: Bernese Alps, Gasteretal; minimumElevationInMeters: 1698; maximumElevationInMeters: 1698; decimalLatitude: 46.4486; decimalLongitude: 7.7438; eventDate: 2011-07-07; habitat: spruce thicket and grass; sex: 1 female; recordedBy: Kuntner, Gregorič, Čandek

Microlinyphia impigra (O. P.-Cambridge, 1871)

\section{Materials}

a. locationID: CH02; country: Switzerland; locality: Bernese Alps, Gasteretal; minimumElevationInMeters: 1698; maximumElevationInMeters: 1698; decimalLatitude: 46.4486; decimalLongitude: 7.7438; eventDate: 2011-07-07; habitat: spruce thicket and grass; sex: 1 females; recordedBy: Kuntner, Gregorič, Čandek

b. locationID: CH23; country: Switzerland; locality: Grison Alps, Alp Flix, Salategnas; minimumElevationInMeters: 1900; maximumElevationInMeters: 1900; decimalLatitude: 46.5141; decimalLongitude: 9.6448; eventDate: 2011-07-12; habitat: forest opening, grass and shrubs; sex: 1 male; recordedBy: Kuntner, Gregorič, Čandek

Page 44: The entries for Palliduphantes pallidus (O. P.-Cambridge, 1871) represent that species and probably Oryphantes angulatus (O. P.-Cambridge, 1881) (new to the checklist) and the corrected text should read:

Palliduphantes pallidus (O. P.-Cambridge, 1871)

Material

a. locationID: $\mathrm{CH03}$; country: Switzerland; locality: Bernese Alps, Gasteretal; minimumElevationInMeters: 1520; maximumElevationInMeters: 1520; decimalLatitude: 46.4498; decimalLongitude: 7.7135; eventDate: 2011-07-07; habitat: spruce forest; sex: 1 male; recordedBy: Kuntner, Gregorič, Čandek 
Oryphantes cf. angulatus (O. P.-Cambridge, 1881)

\section{Material}

a. locationID: CH28; country: Switzerland; locality: Grison Alps, Alp Flix, Salategnas; minimumElevationInMeters: 1713; maximumElevationInMeters: 1713; decimalLatitude: 46.5165; decimalLongitude: 9.6387; eventDate: 2011-07-15; habitat: forest edge; sex: 1 female; recordedBy: Kuntner, Gregorič, Čandek

Page 44: Oedothorax gibbifer (Kulczyn'ski, 1882) is represented with one male and the corrected text should read:

Oedothorax gibbifer (Kulczyn'ski, 1882)

Material

a. IocationID: $\mathrm{CH} 25$; country: Switzerland; locality: Grison Alps, Alp Flix, Salategnas; minimumElevationInMeters: 1950; maximumElevationInMeters: 1950; decimalLatitude: 46.5159; decimalLongitude: 9.6496; eventDate: 2011-07-12/16; habitat: meadow and shrubs at stream; sex: 1 male; recordedBy: Kuntner, Gregorič, Čandek

Page 27, 45: Due to misidentification, Pelecopsis mengei (Simon, 1884) is omitted, and all representatives reported are added to the material listed for Ceratinella brevipes (Westring, 1851) and the corrected text should read:

Ceratinella brevipes (Westring, 1851)

Materials

a. IocationID: $\mathrm{CH} 32$; country: Switzerland; locality: Grison Alps, Alp Flix, Salategnas; minimumElevationInMeters: 1955; maximumElevationInMeters: 1955; decimalLatitude: 46.5203; decimalLongitude: 9.6458; eventDate: 2011-07-16; habitat: timberline forest, moss; sex: 1 male; recordedBy: Kuntner, Gregorič, Čandek

b. locationID: CH22; country: Switzerland; locality: Grison Alps, Alp Flix, Salategnas; minimumElevationInMeters: 1900; maximumElevationInMeters: 1900; decimalLatitude: 46.5152; decimalLongitude: 9.6466; eventDate: 2011-07-12; habitat: forest ground; sex:1 female; recordedBy: Kuntner, Gregorič, Čandek

C. locationID: CH24; country: Switzerland; locality: Grison Alps, Alp Flix, Salategnas; minimumElevationInMeters: 1830; maximumElevationInMeters: 1830; decimalLatitude: 46.5131; decimalLongitude: 9.6430; eventDate: 2011-07-12; habitat: meadow and forest; sex: 1 female; recordedBy: Kuntner, Gregorič, Čandek 
Page 46: Pocadicnemis pumila probably represent that species. The correct number of individuals in material $\mathbf{c}$ is one female and the corrected text in material c should read:

Pocadicnemis cf. pumila (Blackwall, 1841)

Materials

a. locationID: $\mathrm{CH} 11$; country: Switzerland; locality: Bernese Alps, Lake Brienz; minimumElevationInMeters: $\quad 600$; maximumElevationInMeters: 600 ; decimalLatitude: 46.7569; decimalLongitude: 8.0107; eventDate: 2011-07-10; habitat: meadows and forest; sex: 1 female; recordedBy: Kuntner, Gregorič, Čandek

Page 49: The entries for Tenuiphantes jacksonoides (van Helsdingen, 1977) probably represent that species and Tenuiphantes jacksoni (Schenkel, 1925) and the corrected text should read:

Tenuiphantes cf. jacksoni (Schenkel, 1925)

Materials

a. locationID: $\mathrm{CH} 21$; country: Switzerland; locality: Grison Alps, Alp Flix, Salategnas; minimumElevationInMeters: 1970; maximumElevationInMeters: 1970; decimalLatitude: 46.5194; decimalLongitude: 9.6490; eventDate: 2011-07-12; habitat: swamp grazed vegetation; sex: 3 females, 1 male; recordedBy: Kuntner, Gregorič, Čandek

b. locationID: CH28; country: Switzerland; locality: Grison Alps, Alp Flix, Salategnas; minimumElevationInMeters: 1713; maximumElevationInMeters: 1713; decimalLatitude: 46.5165; decimalLongitude: 9.6387; eventDate: 2011-07-15; habitat: forest edge; sex: 1 female; recordedBy: Kuntner, Gregorič, Čandek

Tenuiphantes cf. jacksonoides (van Helsdingen, 1977)

Materials

a. locationID: $\mathrm{CH} 12$; country: Switzerland; locality: Bernese Alps, Nessental; minimumElevationInMeters: 930; maximumElevationInMeters: 930; decimalLatitude: 46.7213; decimalLongitude: 8.3039; eventDate: 2011-07-10; habitat: grassland and lone trees; sex: 1 male; recordedBy: Kuntner, Gregorič, Čandek

b. IocationID: $\mathrm{CH} 22$; country: Switzerland; locality: Grison Alps, Alp Flix, Salategnas; minimumElevationInMeters: 1900; maximumElevationInMeters: 1900; decimalLatitude: 46.5152; decimalLongitude: 9.6466; eventDate: 2011-07-12; habitat: forest ground; sex: 1 female, 4 males; recordedBy: Kuntner, Gregorič, Čandek

c. locationID: $\mathrm{CH} 23$; country: Switzerland; locality: Grison Alps, Alp Flix, Salategnas; minimumElevationInMeters: 1900; maximumElevationInMeters: 1900; 
decimalLatitude: 46.5141; decimalLongitude: 9.6448; eventDate: 2011-07-12; habitat: forest opening, grass and shrubs; sex: 4 females, 3 males; recordedBy: Kuntner, Gregorič, Čandek

d. locationID: $\mathrm{CH} 28$; country: Switzerland; locality: Grison Alps, Alp Flix, Salategnas; minimumElevationInMeters: 1713; maximumElevationInMeters: 1713; decimalLatitude: 46.5165; decimalLongitude: 9.6387; eventDate: 2011-07-15; habitat: forest edge; sex: 6 females; recordedBy: Kuntner, Gregorič, Čandek

Page 54: Due to misidentification, Alopecosa aculeata (Clerck, 1757) is omitted, and the only female reported represents the species Alopecosa taeniata (C. L. Koch, 1835) (new to the checklist) and the corrected text should read:

Alopecosa taeniata (C. L. Koch, 1835)

\section{Material}

a. locationID: CH23; country: Switzerland; locality: Grison Alps, Alp Flix, Salategnas; minimumElevationInMeters: 1900; maximumElevationInMeters: 1900; decimalLatitude: 46.5141; decimalLongitude: 9.6448; eventDate: 2011-07-12; habitat: forest opening, grass and shrubs; sex: 1 female; recordedBy: Kuntner, Gregorič, Čandek

Page 58-59: Due to misidentification, Pardosa cf. hyperborea (Thorell, 1872) is omitted, and the only female reported is added to the material listed for Pardosa oreophila Simon, 1937 and the corrected text should read:

Pardosa oreophila Simon, 1937

Materials

a. locationID: CH10; country: Switzerland; locality: Bernese Alps, Kleine Scheidegg; minimumElevationInMeters: 2061; maximumElevationInMeters: 2061; decimalLatitude: 46.5853; decimalLongitude: 7.9606; eventDate: 2011-07-09; habitat: grassland; sex: 1 female; recordedBy: Kuntner, Gregorič, Čandek

b. locationID: CH13; country: Switzerland; locality: Bernese Alps, Sustenpass; minimumElevationInMeters: 2040; maximumElevationInMeters: 2040; decimalLatitude: 46.7330; decimalLongitude: 8.4324; eventDate: 2011-07-10; habitat: alpine grassland and shrubs; sex: 4 males; recordedBy: Kuntner, Gregorič, Čandek

C. locationID: CH14; country: Switzerland; locality: Glarus Alps, Oberalppass; minimumElevationInMeters: 2040; maximumElevationInMeters: 2040; decimalLatitude: 46.6617; decimalLongitude: 8.6719; eventDate: 2011-07-10; habitat: grassland and shrubs; sex: 1 female; recordedBy: Kuntner, Gregorič, Čandek 
Page 58-59: The entries for Pardosa cf. lugubris (Walckenaer, 1802) represent that species and Pardosa cf. saltans Töpfer-Hofmann, 2000 (new to the checklist) and the corrected text should read:

Pardosa cf. lugubris (Walckenaer, 1802)

Materials

a. locationID: SI15; country: Slovenia; locality: Apače; minimumElevationInMeters: 220; maximumElevationInMeters: 220; decimalLatitude: 46.6804; decimalLongitude: 15.8988; eventDate: 2011-07-26; habitat: forest; sex: 1 female; recordedBy: Kostanjšek, RTŠB 2011

b. IocationID: SI38; country: Slovenia; locality: Poreče; minimumElevationInMeters: 135; maximumElevationInMeters: 135; decimalLatitude: 45.8188; decimalLongitude: 13.9692; eventDate: 2011-05-08; habitat: grassland; sex: 1 female; recordedBy: Čandek

c. IocationID: SI40; country: Slovenia; locality: Slavnik; minimumElevationInMeters: 816; maximumElevationInMeters: 816; decimalLatitude: 45.5499; decimalLongitude: 13.9619; eventDate: 2010-08-26; habitat: grassland and forest; sex: 3 females; recordedBy: Kuntner, Lokovšek

d. locationID: SI58; country: Slovenia; locality: Budanje; minimumElevationInMeters: 243; maximumElevationInMeters: 243; decimalLatitude: 45.8743; decimalLongitude: 13.9497; eventDate: 2011-05-07; habitat: school and surroundings; sex: 2 females; recordedBy: Čandek

e. locationID: SI59; country: Slovenia; locality: Budanje; minimumElevationInMeters: 305; maximumElevationInMeters: 305 ; decimalLatitude: 45.8797 ; decimalLongitude: 13.9468; eventDate: 2011-05-07; habitat: forest; sex: 3 females, 4 males; recordedBy: Čandek

f. locationID: SI60; country: Slovenia; locality: Budanje; minimumElevationInMeters: 295; maximumElevationInMeters: 295; decimalLatitude: 45.8799; decimalLongitude: 13.9459; eventDate: 2011-05-07; habitat: forest clearing; sex: 2 females; recordedBy: Čandek

g. locationID: SI61; country: Slovenia; locality: Sekirišče; minimumElevationInMeters: 750; maximumElevationInMeters: 750; decimalLatitude: 45.8631; decimalLongitude: 14.5367; eventDate: 2011-06-23/2012-06-21; habitat: house, grassland, overgrowth; sex: 4 females; recordedBy: Čandek

Pardosa cf. saltans Töpfer-Hofmann, 2000

\section{Materials}

a. locationID: CH11; country: Switzerland; locality: Bernese Alps, Lake Brienz; minimumElevationInMeters: 600; maximumElevationInMeters: 600; decimalLatitude: 46.7569; decimalLongitude: 8.0107; eventDate: 2011-07-10; habitat: meadows and forest; sex: 3 females; recordedBy: Kuntner, Gregorič, Čandek 
b. locationID: CH12; country: Switzerland; locality: Bernese Alps, Nessental; minimumElevationInMeters: 930; maximumElevationInMeters: 930; decimalLatitude: 46.7213; decimalLongitude: 8.3039; eventDate: 2011-07-10; habitat: grassland and lone trees; sex: 2 females; recordedBy: Kuntner, Gregorič, Čandek

C. locationID: CH15; country: Switzerland; locality: Glarus Alps, near Affeier; minimumElevationInMeters: 817; maximumElevationInMeters: 817; decimalLatitude: 46.7606; decimalLongitude: 9.0933; eventDate: 2011-07-10; habitat: meadow and forest; sex: 4 females; recordedBy: Kuntner, Gregorič, Čandek

d. locationID: CH27; country: Switzerland; locality: Grison Alps, road to Davos; minimumElevationInMeters: 1180; maximumElevationInMeters: 1180; decimalLatitude: 46.6808; decimalLongitude: 9.6557; eventDate: 2011-07-15; habitat: roadside vegetation and forest edge; sex: 6 females, 1 male; recordedBy: Kuntner, Gregorič, Čandek

Page 69-70: The entries for Evarcha laetabunda (C. L. Koch, 1846) represent that species and Evarcha michailovi Logunov, 1992 and the corrected text should read:

Evarcha laetabunda (C. L. Koch, 1846)

Materials

a. IocationID: SI38; country: Slovenia; locality: Poreče; minimumElevationInMeters: 135; maximumElevationInMeters: 135; decimalLatitude: 45.8188; decimalLongitude: 13.9692; eventDate: 2011-05-08; habitat: grassland; sex: 2 females; recordedBy: Čandek

b. IocationID: SI52; country: Slovenia; locality: Dinaric Karst, Griže; minimumElevationInMeters: 484; maximumElevationInMeters: 484; decimalLatitude: 45.7506; decimalLongitude: 13.9509; eventDate: 2011-04-04/05-10; habitat: overgrowth; sex: 1 male; recordedBy: Gregorič, Čandek, Kralj-Fišer

c. IocationID: SI53; country: Slovenia; locality: Dinaric Karst, Griže; minimumElevationInMeters: 434; maximumElevationInMeters: 434; decimalLatitude: 45.7548; decimalLongitude: 13.9495; eventDate: 2011-05-10/2011-06-21; habitat: grassland; sex: 1 female; recordedBy: Gregorič, Čandek

Evarcha michailovi Logunov, 1992

Materials

a. IocationID: SI52; country: Slovenia; locality: Dinaric Karst, Griže; minimumElevationInMeters: 484; maximumElevationInMeters: 484; decimalLatitude: 45.7506; decimalLongitude: 13.9509; eventDate: 2011-04-04/05-10; habitat: overgrowth; sex: 1 male; recordedBy: Gregorič, Čandek, Kralj-Fišer 
b. IocationID: SI56; country: Slovenia; locality: Dinaric Karst, Novelo; minimumElevationInMeters: 358; maximumElevationInMeters: 359; decimalLatitude: 45.8533; decimalLongitude: 13.6552; eventDate: 2011-04-04/05-10; habitat: overgrowth; sex: 5 females, 2 males; recordedBy: Gregorič, Čandek, Kralj-Fišer

c. IocationID: SI53; country: Slovenia; locality: Dinaric Karst, Griže; minimumElevationInMeters: 434; maximumElevationInMeters: 434; decimalLatitude: 45.7548; decimalLongitude: 13.9495; eventDate: 2011-05-10/2011-06-21; habitat: grassland; sex: 1 female; recordedBy: Gregorič, Čandek

Page 72: Misspelling: Leptorchetes should read Leptorchestes

Page 74: Due to misidentification, Pellenes tripunctatus (Walckenaer, 1802) is omitted, and the only male reported is added to the material listed for Pellenes seriatus (Thorell, 1875) and the corrected text should read:

Pellenes seriatus (Thorell, 1875)

Materials

a. IocationID: SI38; country: Slovenia; locality: Poreče; minimumElevationInMeters: 135; maximumElevationInMeters: 135; decimalLatitude: 45.8188 ; decimalLongitude: 13.9692; eventDate: 2011-05-08; habitat: grassland; sex: 1 female; recordedBy: Čandek

b. IocationID: SI52; country: Slovenia; locality: Dinaric Karst, Griže; minimumElevationInMeters: 484; maximumElevationInMeters: 484; decimalLatitude: 45.7506; decimalLongitude: 13.9509; eventDate: 2011-04-04/05-10; habitat: overgrowth; sex: 1 male; recordedBy: Gregorič, Čandek, Kralj-Fišer

c. IocationID: SI53; country: Slovenia; locality: Dinaric Karst, Griže; minimumElevationInMeters: 434; maximumElevationInMeters: 434; decimalLatitude: 45.7548; decimalLongitude: 13.9495; eventDate: 2011-05-10/2011-06-21; habitat: grassland; sex: 1 female; recordedBy: Gregorič, Čandek

d. IocationID: SI55; country: Slovenia; locality: Dinaric Karst, Lokvice; minimumElevationInMeters: 273; maximumElevationInMeters: 275; decimalLatitude: 45.8659; decimalLongitude: 13.6102; eventDate: 2011-04-04/05-10; habitat: overgrowth; sex: 1 female; recordedBy: Gregorič, Čandek, Kralj-Fišer

e. IocationID: SI56; country: Slovenia; locality: Dinaric Karst, Novelo; minimumElevationInMeters: 358; maximumElevationInMeters: 359; decimalLatitude: 45.8533; decimalLongitude: 13.6552; eventDate: 2011-04-04/05-10; habitat: overgrowth; sex: 1 male; recordedBy: Gregorič, Čandek, Kralj-Fišer 
Page 78: The entries for Metellina merianae (Scopoli, 1763) represent that species and Metellina segmentata (Clerk, 1757) and the corrected text should read:

Metellina merianae (Scopoli, 1763)

Materials

a. locationID: CH02; country: Switzerland; locality: Bernese Alps, Gasteretal; minimumElevationInMeters: 1698; maximumElevationInMeters: 1698; decimalLatitude: 46.4486; decimalLongitude: 7.7438; eventDate: 2011-07-07; habitat: spruce thicket and grass; sex: 2 females; recordedBy: Kuntner, Gregorič, Čandek

b. IocationID: SI01; country: Slovenia; locality: Biš; minimumElevationInMeters: 225; maximumElevationInMeters: 225; decimalLatitude: 46.5374; decimalLongitude: 15.8963; eventDate: 2011-07-22; habitat: forest; sex: 1 female; recordedBy: Kostanjšek, RTŠB

c. IocationID: SI29; country: Slovenia; locality: Gradišče pri Lukovici, Gradiško jezero; minimumElevationInMeters: 360; maximumElevationInMeters: 360; decimalLatitude: 46.1626; decimalLongitude: 14.7127; eventDate: 2011-10-06; habitat: lake edge; sex: 2 females, 1 male; recordedBy: Čandek

\section{Metellina segmentata (Clerk, 1757)}

\section{Materials}

a. IocationID: SI36; country: Slovenia; locality: Močilnik; minimumElevationInMeters: 318; maximumElevationInMeters: 318; decimalLatitude: 45.9547; decimalLongitude: 14.2925; eventDate: 2011-10-02; habitat: forest edge; sex: 1 male; recordedBy: Kuntner

b. IocationID: SI50; country: Slovenia; locality: Sp. Prapreče; minimumElevationInMeters: 351; maximumElevationInMeters: 351; decimalLatitude: 46.1620; decimalLongitude: 14.6933; eventDate: 2010-08-03/2012-05-28; habitat: house and surroundings; sex: 1 female, 1 male; recordedBy: Kuntner, Čandek

Page 86: The entries for Robertus lividus (Blackwall, 1836) represent that species and Robertus mediterraneus Eskov, 1987 and the corrected text should read: 
Robertus lividus (Blackwall, 1836)

Material

a. IocationID: $\mathrm{CH} 24$; country: Switzerland; locality: Grison Alps, Alp Flix, Salategnas; minimumElevationInMeters: 1830; maximumElevationInMeters: 1830; decimalLatitude: 46.5131; decimalLongitude: 9.6430; eventDate: 2011-07-12; habitat: meadow and forest; sex: 1 female; recordedBy: Kuntner, Gregorič, Čandek

Robertus mediterraneus Eskov, 1987

Materials

a. locationID: $\mathrm{CH} 15$; country: Switzerland; locality: Glarus Alps, near Affeier; minimumElevationInMeters: 817; maximumElevationInMeters: 817; decimalLatitude: 46.7606; decimalLongitude: 9.0933; eventDate: 2011-07-10; habitat: meadow and forest; sex: 1 male; recordedBy: Kuntner, Gregorič, Čandek

b. IocationID: CH09; country: Switzerland; locality: Pennine Alps, Mattertal; minimumElevationInMeters: 1447; maximumElevationInMeters: 1447; decimalLatitude: 46.0976; decimalLongitude: 7.7789; eventDate: 2011-07-08; habitat: forest and meadow near river; sex: 1 male; recordedBy: Kuntner, Gregorič, Čandek

Page 96: Uloborus walckenaerius Latreille, 1806 is represented with one female and the corrected text should read:

Material

a. IocationID: SI55; country: Slovenia; locality: Dinaric Karst, Lokvice; minimumElevationInMeters: 273; maximumElevationInMeters: 275; decimalLatitude: 45.8659; decimalLongitude: 13.6102; eventDate: 2011-04-04/05-10; habitat: overgrowth; sex: 1 female; recordedBy: Gregorič, Čandek, Kralj-Fišer

Due to less reliable identification the following species should read as corrected:

Page 25: Bolyphantes alticeps (Sundevall, 1833) should read Bolyphantes cf. alticeps (Sundevall, 1833) 
Page 26: Bolyphantes luteolus (Blackwall, 1833) should read Bolyphantes cf. luteolus (Blackwall, 1833)

Page 29: Erigone svenssoni Holm, 1975 should read Erigone cf. svenssoni Holm, 1975

Page 35: Meioneta affinis (Kulczyn'ski, 1898) should read Meioneta cf. affinis (Kulczyn'ski, 1898)

Page 35: Meioneta alpica (Tanasevitch, 2000) should read Meioneta cf. alpica (Tanasevitch, 2000)

Page 35: Meioneta fuscipalpa (C. L. Koch, 1836) should read Meioneta cf. fuscipalpa (C. L. Koch, 1836)

Page 36: Meioneta gulosa (L. Koch, 1869) should read Meioneta cf. gulosa (L. Koch, 1869)

Page 36: Meioneta orites (Thorell, 1875) should read Meioneta cf. orites (Thorell, 1875)

Page 36: Meioneta rurestris (C. L. Koch, 1836) should read Meioneta cf. rurestris (C. L. Koch, 1836)

Page 37: Meioneta saxatilis (Blackwall, 1844) should read Meioneta cf. saxatilis (Blackwall, 1844)

Page 37: Meioneta simplicitarsis (Simon, 1884) should read Meioneta cf. simplicitarsis (Simon, 1884)

Page 46: Pocadicnemis pumila (Blackwall, 1841) should read Pocadicnemis cf. pumila (Blackwall, 1841)

Page 46: Pocadicnemis juncea Locket \& Millidge, 1953 should read Pocadicnemis cf. juncea Locket \& Millidge, 1953

Page 49: Tenuiphantes jacksoni (Schenkel, 1925) should read Tenuiphantes cf. jacksoni (Schenkel, 1925) 
Page 49: Tenuiphantes jacksonoides (van Helsdingen, 1977) should read Tenuiphantes cf. jacksonoides (van Helsdingen, 1977)

New page: Oryphantes angulatus (O. P.-Cambridge, 1881) should read Oryphantes cf. angulatus (O. P.-Cambridge, 1881)

\title{
References
}

- $\quad$ Čandek K, Gregorič M, Kostanjšek R, Frick H, Kropf C, Kuntner M (2013) Targeting a portion of central European spider diversity for permanent preservation. Biodiversity Data Journal 1: e980. DOI: 10.3897/bdj.1.e980

- $\quad$ Coddington J, Young LH, Coyle F (1996) Estimating spider species richness in a southern Appalachian cove hardwood forest. Journal of Arachnology 24: 111-128.

- $\quad$ Coddington J, Agnarsson I, Miller J, Kuntner M, Hormiga G (2009) Undersampling bias: the null hypothesis for singleton species in tropical arthropod surveys. Journal of Animal Ecology 78 (3): 573-584. DOI: 10.1111/j.1365-2656.2009.01525.x

\section{Supplementary materials}

\section{Suppl. material 1: Corrected TaxPub XML of the original publication}

\author{
Authors: Jeremy Miller \\ Data type: XML \\ Brief description: Corrected TaXpub XML of the original publication \\ Filename: BDJ_980_v2.xml - Download file (1.45 MB)
}

\section{Suppl. material 2: Corrected occurrences as Darwin Core Archive}

Authors: Klemen Čandek, Matjaž Gregorič, Rok Kostanjšek, Holger Frick, Christian Kropf, Matjaž Kuntner

Data type: occurences

Filename: occurrences_980_v2.zip - Download file (24.76 kb) 